\title{
5.7 GHz on-chip antenna/RF CMOS transceiver for wireless sensor networks
}

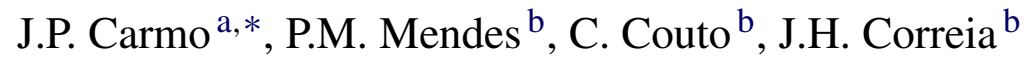 \\ ${ }^{a}$ Polytechnic Institute of Braganca, ESTiG, Campus Santa Apolonia, 5301-857 Braganca, Portugal \\ ${ }^{\mathrm{b}}$ University of Minho, Department of Industrial Electronics, Campus Azurem, 4800-058 Guimaraes, Portugal \\ Received 15 September 2005; accepted 29 March 2006 \\ Available online 20 July 2006
}

\begin{abstract}
This paper describes a chip-size antenna for operation at $5.7 \mathrm{GHz}$, assembled with a low-power, low-voltage RF CMOS transceiver, fabricated in UMC RF CMOS $0.18 \mu \mathrm{m}$ process. Measurements shown a patch antenna with the central frequency of $5.705 \mathrm{GHz}$, a bandwidth of $90 \mathrm{MHz}$ at $-10 \mathrm{~dB}$ of return loss, a directive gain of $0.3 \mathrm{~dB}$, with an efficiency of $18 \%$, and a transceiver with a measured total power consumption of $23 \mathrm{~mW}$. This microsystem is intended for the use in each wireless node of a wireless sensor network mounted in a wireless electronic shirt, that monitors the cardio-respiratory function and posture.
\end{abstract}

(C) 2006 Elsevier B.V. All rights reserved.

Keywords: Wireless sensors networks; On-chip antenna; RF transceiver

\section{Introduction}

Wireless communication microsystems with high density of nodes and simple protocol are emerging for low-data-rate distributed sensor network applications such as those in home automation and industrial control [1].

It is available a huge range of solutions, concerning the implementation of wireless sensors networks. A few companies [2-4] are offering products such as radios (motes) and sensor interfaces. The motes are battery-powered devices that run specific software. In addition to running the software networking stack, each mote can be easily customized and programmed, since it runs open-source operating systems which provides low-level event and task management. Mote Processor/Radio module families working at $2.4 \mathrm{GHz}$ ISM band and supporting IEEE802.15.4 and ZigBee are available [2-4].

However, the implementation of a wireless bus in certain applications requires compact and miniaturized solutions. Moreover, a chip-size antenna included in the RF microsystem will be crucial.

\footnotetext{
* Corresponding author. Tel.: +351 273 303000; fax: +351 273313051. E-mail address: jcarmo@ipb.pt (J.P. Carmo).
}

This type of wireless microsystem with sensors, electronics and antenna has interest for electronic textiles as the application presented in this paper. Moreover, in order to implement an efficient power-consumption wireless sensor network in clothes (e.g. an wireless electronic shirt), it is necessary to develop a low-power low-voltage RF transceiver, mounted with a patch antenna. As the operating frequencies are increasing (the IEEE802.11a is an example), on-chip antenna integration with RF CMOS transceivers with reasonable efficiency becomes feasible. Moreover, due to the frequency increase, the provided bandwidth becomes also acceptable both for data communications and sensor applications. The target application of this RF microsystem is the implementation of a wireless sensors network in a wireless electronic shirt (WES) for monitoring the cardio-respiratory function and posture.

\section{RF CMOS transceiver at $5.7 \mathrm{GHz}$}

\subsection{Design}

It was fabricated a RF CMOS transceiver operating at $5.7 \mathrm{GHz}$ ISM band, with ASK modulation. The UMC RF $0.18 \mu \mathrm{m}$ CMOS process allows to trade the high-frequency capability of minimum-length transistors with lower current consumption by biasing the devices at lower current densities, even 


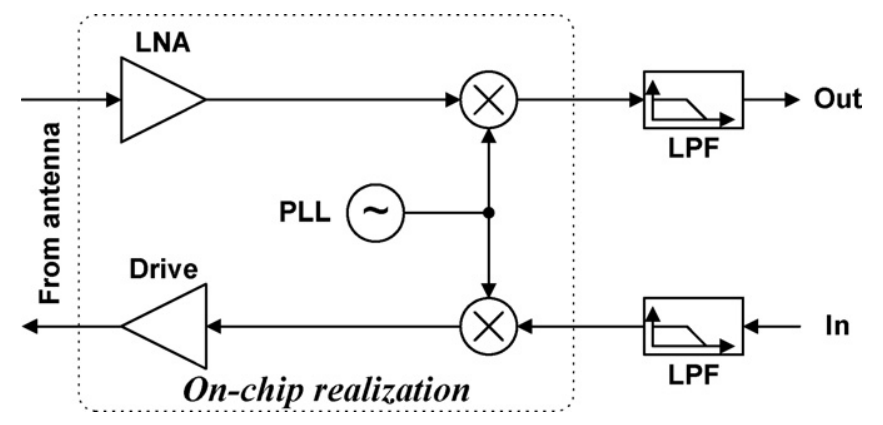

Fig. 1. The RF transceiver structure.

for devices working at RF. This process provides a poly and six metal layers, the use of integrated spiral inductors (with a quality factor of 10), high-resistor values (a special layer is available) and a low power supply of $1.5 \mathrm{~V}$. The transceiver has a lownoise amplifier (LNA) that provides a $50 \Omega$ input impedance, the amplified RF signal is directly converted to the baseband with a single balanced active MOS mixer. The internal oscillator is a phase-locked loop (PLL) working at 5.7 GHz. The whole transceiver structure is illustrated in Fig. 1.

The transceiver is able to operate at the $[5.420-5.8265 \mathrm{GHz}]$ frequency range. This is done by changing the frequency division ratio in the feedback path of the PLL. The PLL has four digital inputs for the division ratio programming. The output frequency is

$f_{\text {out }}=f_{\text {ref }} \times 2 \times(200+D)$

where $D$ is the decimal representation of the division ratio. The used reference frequency was $f_{\text {ref }}=13.56 \mathrm{MHz}$.

In high frequency PLLs, the high power consumption is mainly due to the first stages of the frequency divider that often dissipates half of the total power. The use of conventional static CMOS logic in the first stage is not possible. This is due to the high input frequency [5]. The overall divider has three truesingle-phase-clock (TSPC) frequency dividers, that halves the following dividers, which use static logic.

In order to have the lowest noise figure (NF), the LNA is an inductively degenerated common source amplifier with tuned load. This makes the input impedance at $5.7 \mathrm{GHz}$ equal to 50 (, for matching with antenna. As depicted in Fig. 2, it was used a single transistor in the amplifier. The reduction of active devices sacrifices the gain, but achieves lowest NFs.

It was used for the up and for the downconversion frequency, $I-Q$ mixers that are ac-coupled to the LNA and are based on the modified Gilbert cell (Fig. 3) [6]. The mixers are directly driven by the differential outputs of the on-chip frequency synthesizer.

\subsection{Results}

At frequencies in the range [5.420-5.8265 GHz], the measurements show for the LNA a gain in the range [9.597-9.807 dB], a NF in the range [0.775-0.841 dB] and a stabilization factor $K$ of 1.209 , making the LNA unconditionally stable $(K>1)$. Measurements show for the LNA, a power consumption of $9.65 \mathrm{~mW}$. The power consumptions are about of

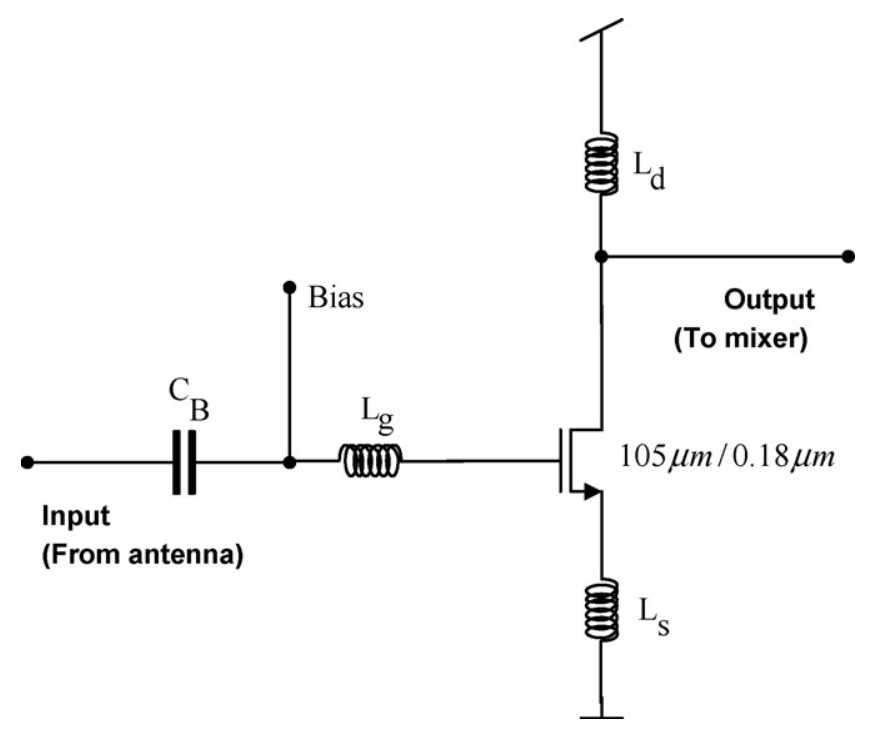

Fig. 2. The low-noise-amplifier schematic.

$9.51 \mathrm{~mW}$ for the mixers, and $4.14 \mathrm{~mW}$ for the PLL. A photo of the transceiver used for the transmission at $5.7 \mathrm{GHz}$ is depicted in Fig. 4.

\section{Antenna}

\subsection{Design}

Different solutions have been suggested to achieve antenna integration within a single chip. Since high losses of standardresistivity silicon are prohibitive for antenna integration, most of the proposed solutions rely on high-resistivity silicon (HRS) or micromachined substrates. The HRS solution uses a bulk substrate having the same electrical permittivity but lower losses. In micromachined substrates, the losses are reduced by selective substrate removal underneath the metal patch. The drawback is

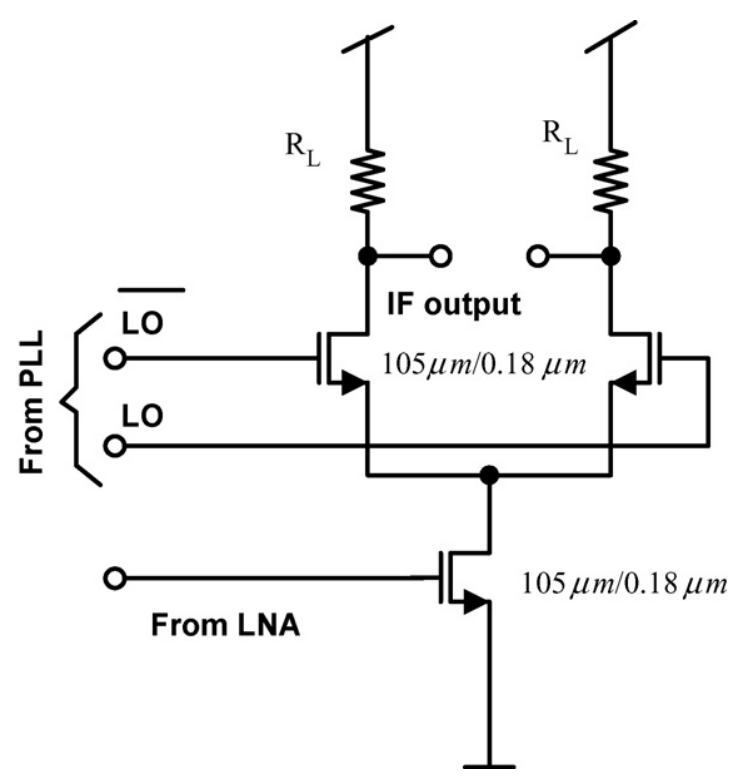

Fig. 3. The mixer schematic 


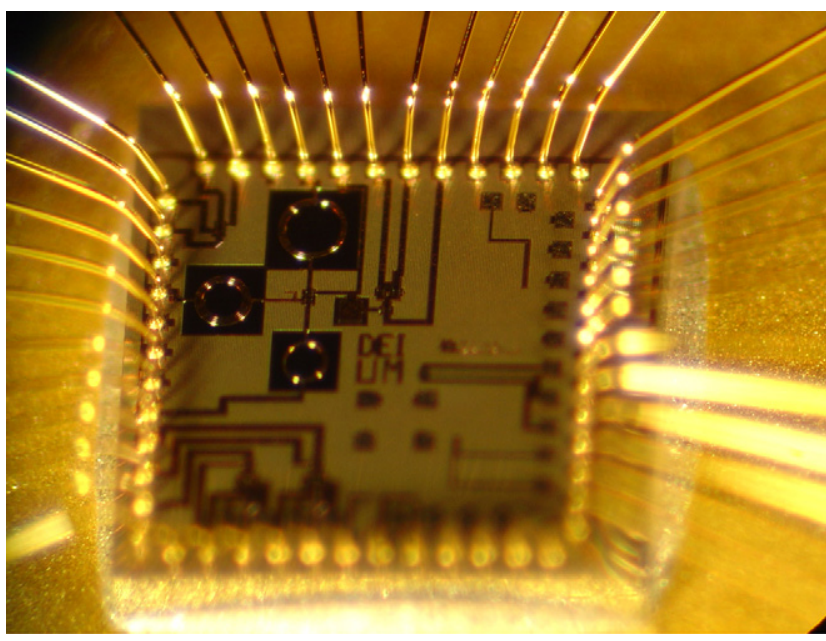

Fig. 4. A photo of the transceiver used for the transmission at $5.7 \mathrm{GHz}$.

an increase of antenna size due to the effective electrical permittivity reduction resulting from the partial replacement of silicon by air. It was used for the substrate, high-resistivity silicon (HRS) together with insulations layers to keep the losses at low as possible. The HRS substrate has a dielectric permittivity of 11.7, conductivity in the range $0.02-0.05 \mathrm{~S} / \mathrm{m}$, and the wafer thickness of $525 \pm 25 \mu \mathrm{m}$. The use of HRS is enough to provide considerable loss reduction. Nevertheless, the losses can be reduced even further with the use of a dioxide layer between the silicon wafer and the metal patch. This layer has a permittivity of 3.9 and is an insulator. The ground and metal patches were made of aluminum, with a thickness of $2 \mu \mathrm{m}$. Fig. 5 illustrates the materials and configuration used in the fabrication. Antenna feeding was carefully designed, in order to provide a correct input impedance (50 () to do the measurements. A photo of the fabricated patch antenna prototype, with $7.7 \mathrm{~mm} \times 7.6 \mathrm{~mm}$ area dimensions is illustrated in Fig. 6.

\subsection{Results}

A $8510 \mathrm{C}$ vector network analyzer was used to measure the return loss. The measured values for the antenna using HRS substrate are plotted against the simulated data in Fig. 7. It can be seen that the simulated data agrees quite well with the measured. The obtained operating frequency was near to $5.705 \mathrm{GHz}$ and the $-10 \mathrm{~dB}$ return loss bandwidth was $90 \mathrm{MHz}$.

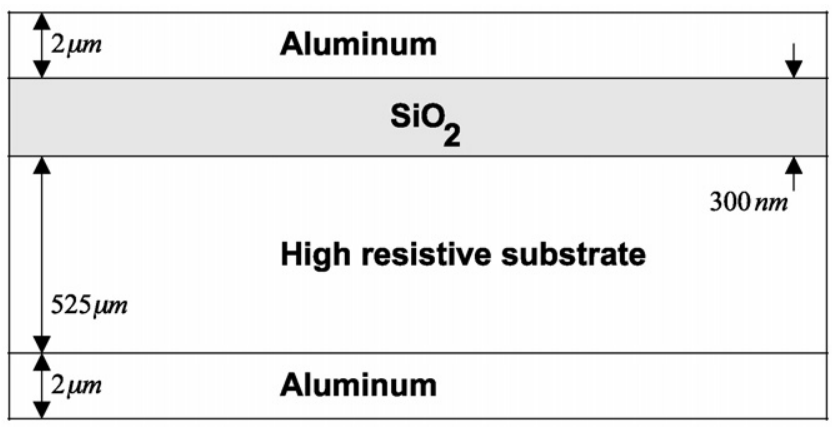

Fig. 5. Cross-section of the patch antenna design in HRS wafer.

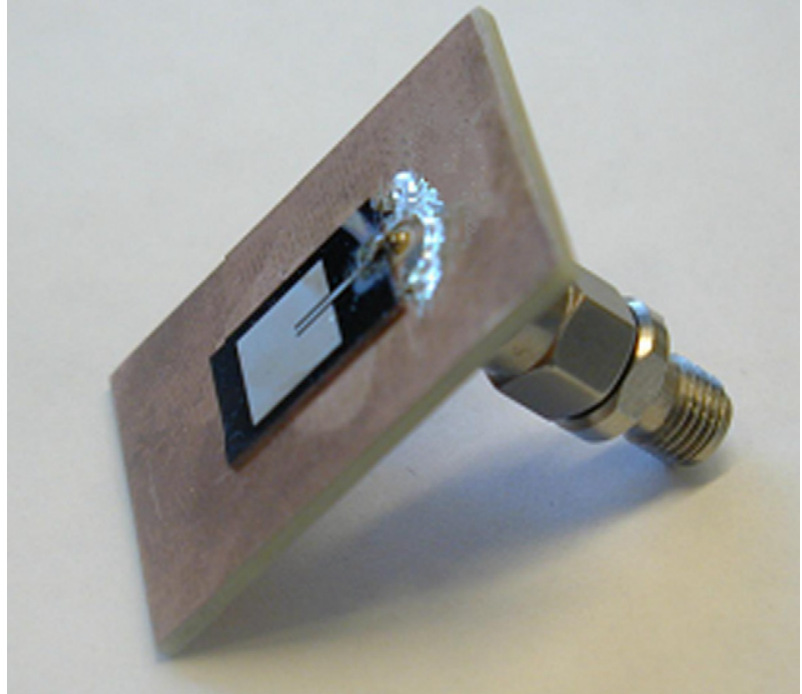

Fig. 6. A photo of the patch antenna fabricated on an HRS wafer, mounted on an SMA connector for measurements.

\section{Wireless electronic shirt application}

\subsection{Introduction}

Today, the link between textiles and electronics is more realistic than ever. An emerging new field of research that combines the strengths and capabilities of electronics and textiles into one: electronic textiles, or e-textiles is opening new opportunities. E-textiles, also called smart fabrics, have not only wearable capabilities like any other garment, but also have local monitoring and computation, as well as wireless communication capabilities. Sensors and simple computational elements are embedded in e-textiles, as well as built into yarns, with the goal of gathering sensitive information, monitoring vital statistics, and sending them remotely over a wireless channel, for further processing [7]. For integration into everyday clothing, electronic components should be designed in a functional, unobtrusive, robust, small, and inexpensive way. Therefore, small single-chip micro-

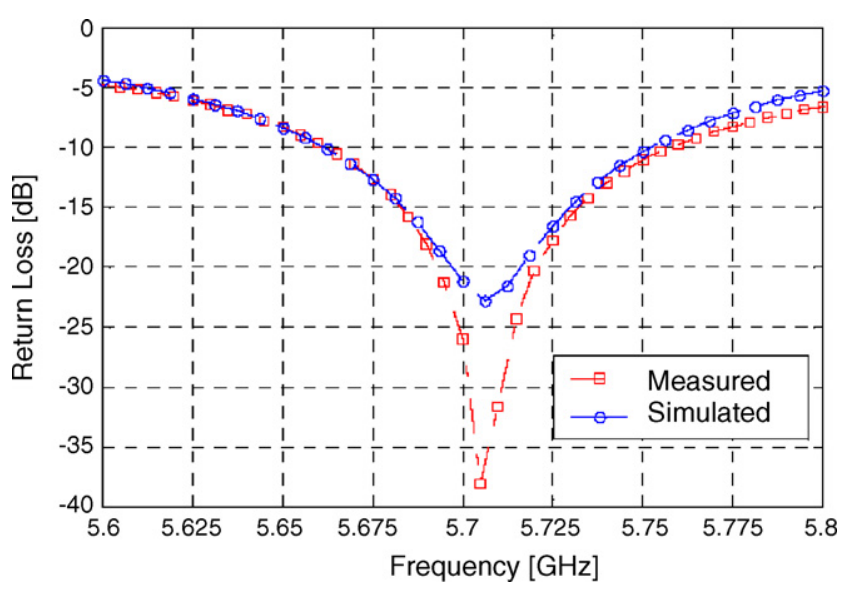

Fig. 7. Measured and simulated return loss for the antenna using HRS as substrate. 
electronic systems rather than large-scale computer boxes are a promising approach.

The electronic shirt's goal is the monitoring of the cardiorespiratory function. This makes it able to recognize qualitatively and quantitatively the presence of respiratory disorders, both during wake and sleep-time in free-living patients with chronic heart failure, providing clinical and prognostic significance data $[8,9]$.

In traditional e-textile, the communication among processing elements is wired [7]. Generally, a flexible data bus integrated into the structure, is used to route the information from the sensors to the controller $[10,11]$. The wired concept is a problem when the textiles going to wash, because require removal of complex electronics before starting the cleaning process. Another disadvantage, is that the topological location of different processing elements is fixed throughout the lifetime of the applications.

\subsection{Wireless electronic shirt concept}

Like any other every-day garment piece, the WES will be lightweight, machine washable, comfortable, easy-to-use shirt with embedded sensors. To measure respiratory and cardiac functions, sensors are plugged into the shirt around patient's chest and abdomen. A single channel measures heart rate and a MEMS capacitive accelerometers network records patient posture (arms and body position) and activity level (arms movements). Inductive cooper filaments will be used for monitoring the respiratory function. It is used the commercial FLX-01 sensor to monitor the shoulders positions [12]. The FLX-01 has changes in the electrical resistance when it is bent. As the FLX01 sensor is bent the resistance gradually increases. When the sensor is bent at $90^{\circ}$, its resistance will range between from the $30 \mathrm{k} \Omega$ through the $40 \mathrm{k} \Omega$. It was also used the MLT1132 Piezo Respiratory Belt Transducer [13] to measure the changes in thoracic or abdominal circumference due to respiration. The transducer contains a sensor placed between two elastic strips that measures changes in abdominal or thoracic circumference. Stretching of the elastic places a strain on the piezo-sensor, which generates a voltage. The device should be placed around the body at the level of maximum respiratory expansion. This level will change between erect and supine positions. At maximum inspiration the belt should be stretched almost to maximum extension. This allows the recording of respiratory changes with maximum sensitivity and linearity.

It is shown in Fig. 8 a photo of the patient wearing an WES ready to plug the RF microsystem (antenna + transceiver). It can be seen the three connections for heart-rate respiratory function and posture.

In WES, the sensor interfaces, data processing, the wireless interface and antenna are integrated in the same microsystem by multi-chip-module techniques. The wireless communication is between the base-station and the multiple processing elements placed in the shirt. The main advantage is to allow the positioning of the sensors where we like. The sensors can also be removed from the shirt, either when the sensors are no more need or when the shirt is to be cleaned and washed. This wireless bus

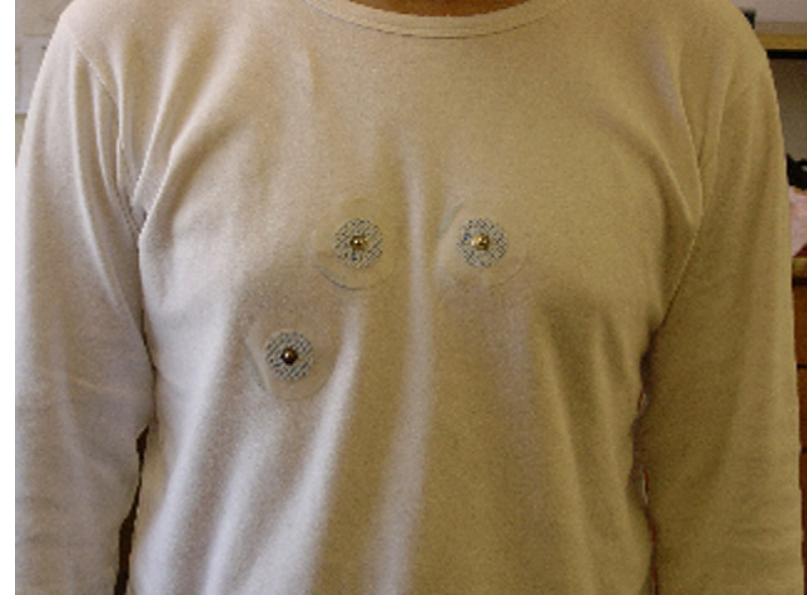

Fig. 8. A photo of the patient wearing a electronic shirt ready to plug the RF microsystem (antenna + transceiver). We can see the three connections for heart rate with a single electrode, respiratory function and posture.

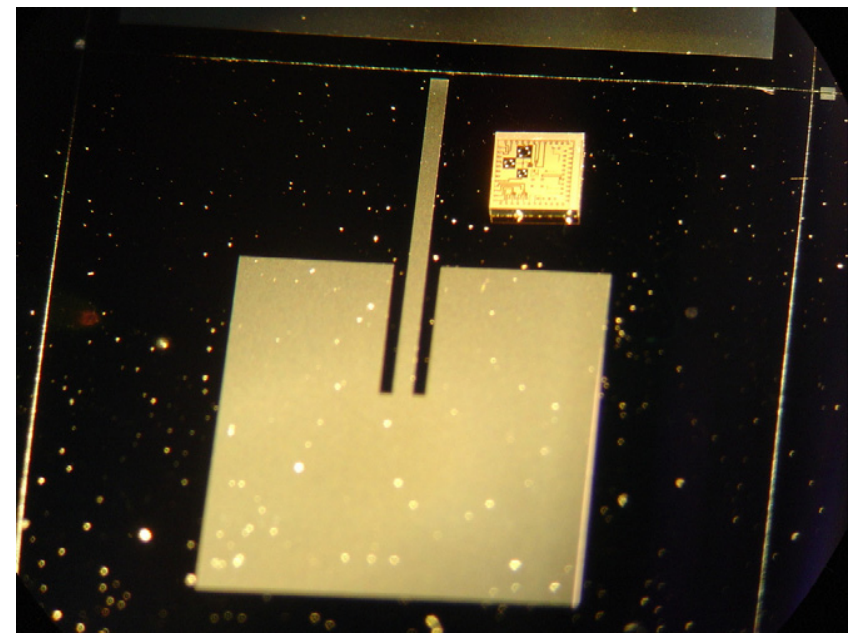

Fig. 9. Chip-size antenna for operation at $5.7 \mathrm{GHz}$ assembled with an RF CMOS transceiver.

introduces the concept of plug-and-play in textiles. Fig. 9 shows a photo of the chip-size antenna for operation at $5.7 \mathrm{GHz}$ assembled with a RF CMOS transceiver.

\section{Conclusions}

WES allows patients to wear electronic devices and maintain their mobility while simultaneously having their cardiorespiratory function and posture monitored.

A chip-size antenna for operation at $5.7 \mathrm{GHz}$, assembled with a low-power, low-voltage RF CMOS transceiver was presented in this paper. The microstrip patch antenna fabricated on HRS has area of $8 \mathrm{~mm}^{2}$ and operates at $5.705 \mathrm{GHz}$ with approximately $90 \mathrm{MHz}$ of bandwidth and a gain of $0.3 \mathrm{~dB}$. The measurements show a total power consumption of $23 \mathrm{~mW}$, for the transceiver. This microsystem is intended to the implementation of wireless nodes in a wireless sensors network mounted in an electronic shirt that monitors the cardio-respiratory functions. It was also presented a new type of wireless bus, where the RF interfaces 
containing sensors can be added and removed without special care concerning the wireless network management. In WES it was introduced a new technological concept: the plug-and-play modules in textiles.

\section{References}

[1] P. Choi, H. Park, S. Kim, S. Park, I. Nam, T.W. Kim, S. Park, S. Shin, M.S Kim, K. Kang, Y. Ku, H. Choi, S.M. Park, K. Lee, An experimental coinsized radio for extremely low-power WPAN (IEEE 802.15.4) application at $2.4 \mathrm{GHz}$, IEEE J. Solid-State Circuits 38 (12) (2003) 2258-2268.

[2] Crossbow Inc., http://www.xbow.com/.

[3] Dust Networks Inc., http://www.dust-inc.com/.

[4] Sensicast Systems, http://www.sensicast.com/.

[5] S. Pellerano, S. Levantino, C. Samori, A.L. Lacaita, A 13.5-mW 5-GHz frequency synthesizer with dynamic-logic-frequency divider, IEEE J. SolidState Circuits 39 (2) (2004) 378-383.

[6] G. Montagna, G. Gramegna, I. Bietti, M. Franciotta, A. Barschirotto, P. De Vita, R. Pelleriti, M. Paparo, R. Castello, A 35-mW 3.6- $\mathrm{mm}^{2}$ fully integrated 0.18- $\mu \mathrm{m}$ CMOS GPS radio, IEEE J. Solid-State Circuits 39 (7) (2004) 1163-1171.

[7] D. Marculescu, R. Marculescu, N.H. Zamora, P. Stanley-Marbell, P.K. Khosla, S. Park, S. Jayaraman, S. Jung, C. Lautervach, W. Weber, T. Kirstein, D. Cottet, J. Grzyb, G. Tröster, M. Jones, T. Martin, Z. Nakad, Electronic textiles: a platform for pervasive computing, Proc. IEEE 91 (12) (2003) 1995-2018.

[8] J.H. Correia, P.M. Mendes, Wireless hydrotherapy smart suit for monitoring handicapped people, in: Proceedings of the 2004 SPIE Conference in Smart Materials, Nano- and Micro-Smart Systems, Sydney, Australia, December 12-15, 2004, pp. 385-392.

[9] J.P. Carmo, P.M. Mendes, C. Couto, J.H. Correia, $2.4 \mathrm{GHz}$ wireless sensor network for smart electronic shirts, in: Proceedings of the Microtechnologies for the New Millennium 2005, Sevilha, Spain, May 9-11, 2005, pp. 579-586.

[10] Sensatex Inc., http://www.sensatex.com/.

[11] J. Coosemans, B. Hermans, R. Puers, Integrating wireless ECG monitoring in textiles, in: Proceedings of the Transducers 2005, Seoul, South Korea, June 5-9, 2005, pp. 228-232.
[12] FLX-01 Flexible Bend Sensor, http://www.imagesco.com/.

[13] MLT1132 Piezo Respiratory Belt Transducer, http://www.adinstruments. $\mathrm{com} /$.

\section{Biographies}

J.P. Carmo graduated in 1993 and received his MSc degree in 2002, both in Electrical Engineering and Computers from the University of Oporto, Oporto, Portugal. Since 1999, he is a lecturer at the Polytechnic Institute of Bragança, Portugal. He is currently working towards his $\mathrm{PhD}$ degree in Industrial Electronics and is involved in the research on wireless microsystems.

P.M. Mendes graduated in 1995 and received his MSc degree in 1999, both in Electrical Engineering from the University of Coimbra, Coimbra, Portugal. From 1997 to 1999, he was a lecturer at the Polytechnic Institute of Castelo Branco, Portugal. Since 1999, he has been a lecturer at the Department of Industrial Electronics, University of Minho, Portugal. He is currently working towards his $\mathrm{PhD}$ degree in Industrial Electronics and is involved in the research on waferlevel chip-scale packaging for RF applications and wireless microsystems.

C. Couto graduated in Electrical Engineering at University of Lourenco Marques, Mozambique in 1972. He obtained the MSc degree in 1979 and $\mathrm{PhD}$ degree in 1981 at UMIST (University of Manchester Institute of Science and Technology), UK, both in power electronics. In 1976, he joined the University of Minho in Portugal, where since 1995, he has been full professor in the Department of Industrial Electronics. His research interests are microsystems, instrumentation and power electronics.

J.H. Correia graduated in Physical Engineering from University of Coimbra, Portugal in 1990. He obtained in 1999 a PhD degree at the Laboratory for Electronic Instrumentation, Delft University of Technology, working in the field of microsystems for optical spectral analysis. Presently, he is an Associate Professor in Department of Industrial Electronics, University of Minho, Portugal. He was the General-Chairman of Eurosensors 2003, Guimaraes, Portugal. His professional interests are in micromachining and microfabrication technology for mixed-mode systems, solid-state integrated sensors, microactuators and microsystems. 